\title{
Syndrome de Guillain-Barré consécutif à la vaccination contre le SRAS-CoV-2 chez un patient ayant déjà présenté ce syndrome en lien avec un vaccin
}

\author{
Ling Ling MD, Sean M. Bagshaw MD MSc, Pierre-Marc Villeneuve MD MSc
}

— Citation : CMAJ 2021 November 22;193:E1766-9. doi : 10.1503/cmaj.210947-f

Voir la version anglaise de l'article ici : www.cmaj.ca/lookup/doi/10.1503/cmaj.210947

$\mathbf{U}$ n homme de 63 ans a consulté aux urgences pour faiblesse ascendante progressive aux bras et aux jambes. Le patient avait des antécédents d'hypertension, de dyslipidémie, de diabète sucré insulinodépendant, de néphrolithiase, d'obésité, d'anxiété et de syndrome de Guillain-Barré (SGB) consécutif à un vaccin antigrippal. Ses médicaments étaient insuline glargine, metformine, sémaglutide, gliclazide, amlodipine, valsartan, atorvastatine et citrate de potassium.

En septembre 1999, environ 3 semaines après avoir reçu un vaccin contre la grippe saisonnière, le patient a présenté une paralysie faciale aiguë, une faiblesse et une paresthésie ascendantes aux membres supérieurs et inférieurs, progressant vers une paralysie flasque des 4 membres accompagnée d'aréflexie généralisée. La tomodensitométrie n'a révélé aucune anomalie cérébrale à ce moment. La ponction lombaire a révélé des taux élevés de protéines dans le liquide céphalorachidien (LCR). Aucun test de conduction nerveuse n'a été effectué, mais le diagnostic de SGB a été posé sur la base du tableau clinique et des résultats d'analyses. Le patient a eu besoin d'une ventilation mécanique prolongée et d'une trachéotomie et il a été traité au moyen de corticostéroïdes systémiques et d'immunoglobulines intraveineuses. Il a fini par se rétablir presque complètement sur le plan neurologique, ne conservant qu'une légère paralysie faciale résiduelle. Enfant, le patient avait reçu ses vaccins sans effets indésirables; c'était son premier vaccin antigrippal, et il n'avait reçu aucun autre vaccin jusqu'à la pandémie causée par le virus SRAS-CoV-2.

Douze jours avant sa consultation aux urgences, il a reçu sa première dose de vaccin ChAdOx1 nCoV-19 (AstraZeneca), un vaccin à adénovirus simien non réplicatif contre le SRAS-CoV-2. II a eu de légers frissons et de la fatigue, qui sont rentrés dans l'ordre en l'espace de 3 jours après la vaccination. Onze jours plus tard, il a présenté une paresthésie des lèvres et des doigts des 2 mains. Le jour où il a consulté, il a rapidement éprouvé une faiblesse

\section{Points clés}

- De très rares cas de syndrome de Guillain-Barré (SGB) ont été recensés après une vaccination, mais aucun lien causal n'a formellement été établi.

- Le SGB pourrait être considéré dans le diagnostic différentiel chez un patient qui consulte pour faiblesse musculaire ascendante symétrique avec déficit sensoriel après une vaccination contre la grippe ou le SRAS-CoV-2.

- Un nombre plus grand de cas de SGB ont été signalés après l'infection au SRAS-CoV-2 qu'après la vaccination; les avantages de la vaccination contre le SRAS-CoV-2 en surclassent les effets indésirables potentiels chez la quasi-totalité des patients.

- Les risques et avantages de la vaccination contre le SRAS-CoV-2 doivent faire l'objet d'une discussion approfondie avec les patients qui ont déjà connu un épisode de SGB après avoir reçu un vaccin.

ascendante progressive des bras et des jambes. Il n'avait eu jusqu'alors aucun symptôme pouvant suggérer une infection.

À l'arrivée, l'examen physique a montré une légère faiblesse (4+/5, selon l'échelle du MRC [British Medical Research Council] pour la force musculaire) des abducteurs des épaules et des fléchisseurs des genoux, même si la force du patient était par ailleurs préservée. Il avait une aréflexie, une perte bilatérale de la sensibilité aux piqûres d'aiguille et à la vibration jusqu'à mijambe, sans niveau sensoriel, et une perte de proprioception jusqu'à l'articulation de la cheville. Ses enjambées étaient anormalement grandes. Après son admission, sa faiblesse a progressé et atteint $1 / 5$ pour les dorsifléchisseurs des chevilles, $1 / 5$ pour les longs extenseurs de l'hallux, 3-4/5 pour les extenseurs des genoux, $1-2 / 5$ pour les fléchisseurs des hanches et $4 / 5$ pour les abducteurs des épaules, les fléchisseurs et extenseurs des coudes et les extenseurs des poignets, et ce, des 2 côtés. 
Les examens électrophysiologiques ont montré une neuropathie périphérique sensorimotrice avec démyélinisation substantielle et caractéristiques axonales surimposées concordant avec un diagnostic de SGB (figure 1 et tableau 1). L'analyse du LCR du patient a montré une dissociation albinocytologique (c.-à-d. hausse des protéines sans leucocytose), avec numération leucocytaire acceptable de 1 (normale $\leq 5) \times 10^{6} / \mathrm{L}$, taux de protéines élevé de 0,6 (normale $0,15-0,45) \mathrm{g} / \mathrm{L}$, augmentation du taux de glucose à 6,6 (normale 2,2-4,4) $\mathrm{mmol} / \mathrm{L}$ (glycémie de $11,5 \mathrm{mmol} / \mathrm{L}$ ) et de lactate à 2,8 (normale $<2,8$ ) $\mathrm{mmol} / \mathrm{L}$. Nous n'avons trouvé aucun signe d'infection ni cause métabolique ou structurelle à ses symptômes. Plus précisément, les cultures bactériennes du LCR ont été négatives, tout comme la recherche d'acides nucléiques d'entérovirus, de paréchovirus, de virus de la varicelle et du zona et de virus de l'herpès simplex de types 1 et 2 . Les analyses sériques ont confirmé l'absence d'hépatite $\mathrm{B}$, d'hépatite $\mathrm{C}$, de VIH et de syphilis. Les hémocultures étaient stériles. La fonction thyroïdienne et surrénalienne et les taux d'électrolytes étaient normaux. Une tomodensitométrie de la tête du patient n'a montré aucune anomalie aiguë, même si nous avons observé une maladie ischémique microvasculaire en arrièreplan. L'imagerie par résonance magnétique de la colonne cervicale n'a révélé aucune anomalie de la moelle épinière cervicale. Notre centre ne procède pas systématiquement à la recherche des autoanticorps antigangliosides neuronaux sériques dans les cas typiques de SGB, et cette analyse n'a pas été effectuée dans le cas présent.
Nous avons d'abord traité le patient au moyen d'immunoglobulines intraveineuses. Toutefois, comme sa faiblesse motrice s'accentuait et sa capacité vitale se détériorait, nous avons procédé à un échange plasmatique thérapeutique. En plus des soins de soutien habituels, le patient a eu besoin de ventilation mécanique non effractive pendant 3 jours, mais n'a pas été placé sous ventilation mécanique effractive.

Trois mois après la consultation initiale, la fonction motrice de notre patient s'était substantiellement améliorée. Il continuait de présenter une légère faiblesse (4/5) aux mains et aux portions proximales et distales des membres inférieurs, une aréflexie ainsi qu'une anomalie de la sensation du toucher léger, des piqûres d'aiguille et de la vibration.

Le service d'immunologie et d'allergologie cliniques demandé en consultation a indiqué qu'il n'y avait aucun test in vitro permettant d'identifier avec certitude un quelconque déclencheur de l'épisode de SGB. Le lien temporel entre la vaccination et le déclenchement des symptômes 12 jours plus tard était attendu, puisque le SGB n'est pas médié par l'immunoglobuline E (IgE), comme l'anaphylaxie.

Nous avons suggéré au patient d'éviter toute autre dose du vaccin ChAdOx1 nCoV-19. On dispose de peu de données probantes pour formuler une recommandation chez ce patient en ce qui concerne l'innocuité des vaccins contre le SRAS-CoV-2 qui n'utilisent pas de vecteurs viraux.

\section{A Moteur}
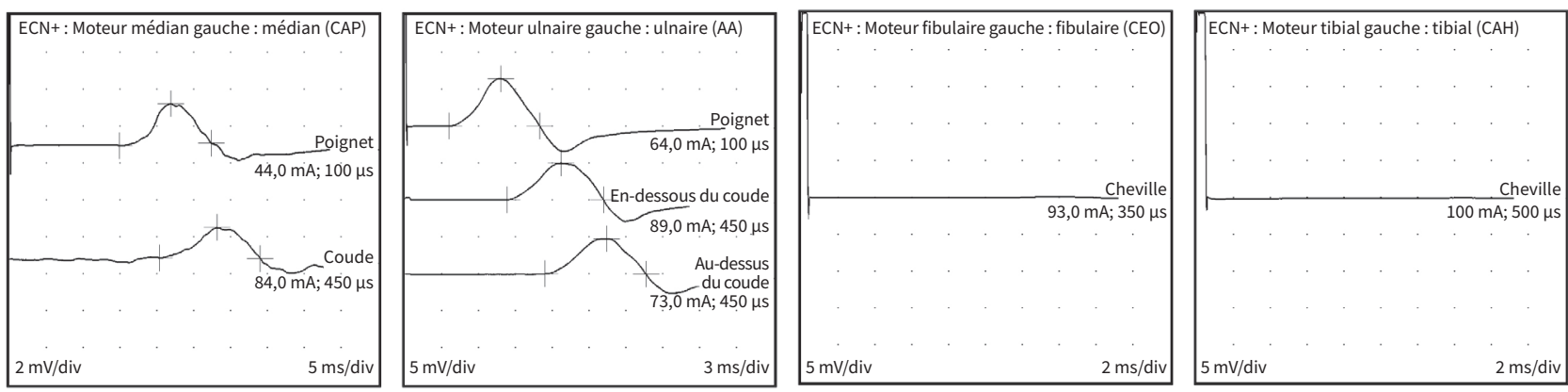

\section{B Sensoriel}
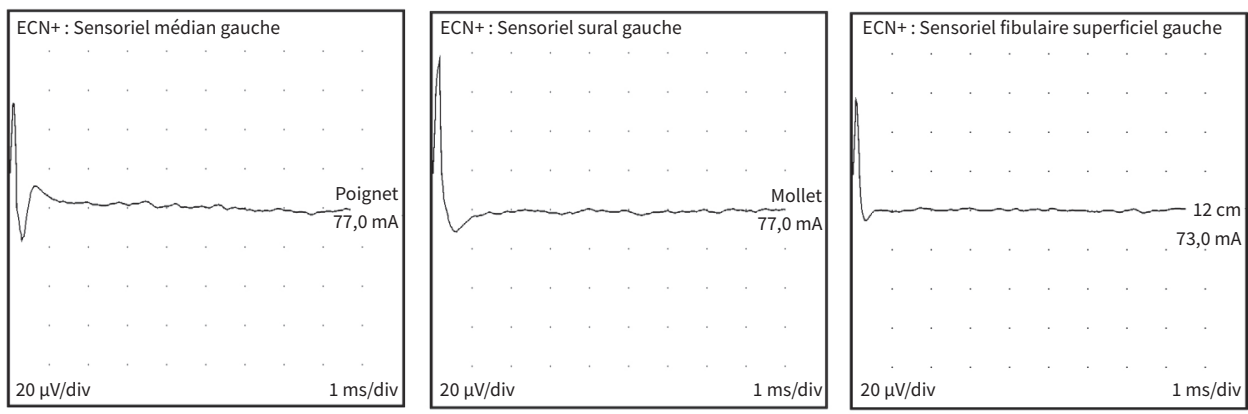

Figure 1 : Ondes enregistrées lors des examens de la conduction nerveuse motrice (A) et sensorielle (B) chez un homme de 63 ans présentant un syndrome de Guillain-Barré (SGB). Ces résultats sont indicateurs du SGB et comportent des caractéristiques axonales et démyélinisantes typiques, telles que la vitesse et l'amplitude réduites. Le déficit moteur était plus prononcé au niveau des nerfs de la fibula (péroné) et du tibia gauches, comme le montre l'absence totale de conduction. Un déficit sensoriel sévère était également présent au niveau des nerfs sensitifs étudiés. Remarque : AA = abducteur de l'auriculaire, $\mathrm{CAH}=$ court abducteur de l'hallux, $\mathrm{CAP}=$ court abducteur du pouce, $\mathrm{CEO}=$ court extenseur des orteils, $\mathrm{ECN}+=$ examen de la conduction nerveuse, $\mathrm{ms} / \mathrm{div}=$ milliseconde par division, $\mathrm{mV} / \mathrm{div}=$ millivolt par division . 
Tableau 1 : Examens de la conduction nerveuse chez un homme de 63 ans atteint du syndrome de Guillain-Barré*

\begin{tabular}{|c|c|c|c|c|c|c|}
\hline Sites & Latence, ms & Amplitude, mV & $\begin{array}{c}\text { Zone } \\
\text { négative, } \\
\text { ms*mv }\end{array}$ & $\begin{array}{l}\text { Latence } \\
\text { minimum de } \\
\text { l'onde F, ms }\end{array}$ & Distance, $\mathrm{cm}$ & $\mathrm{CV}, \mathrm{m} / \mathrm{s}$ \\
\hline \multicolumn{7}{|l|}{ Sites moteurs } \\
\hline \multicolumn{7}{|l|}{ Médian gauche (CAP) } \\
\hline Poignet & 14,9 & 2,3 & 13,7 & AR & 7 & \\
\hline Coude & 20,4 & 1,67 & 11,5 & & 23 & 42 \\
\hline \multicolumn{7}{|l|}{ Ulnaire gauche $(A A)$} \\
\hline Poignet & 3,6 & 6,4 & 23,5 & 37,6 & 6 & \\
\hline En dessous du coude & 8,3 & 5,0 & 21,8 & & 27 & 57 \\
\hline Au-dessus du coude & 11,4 & 4,8 & 21,5 & & 11 & 35 \\
\hline \multicolumn{7}{|l|}{ Radial gauche (EPI) } \\
\hline Avant-bras & 2,9 & 5,4 & 26,9 & & 8 & \\
\hline Bras & 8,9 & 3,8 & 17,9 & & 26 & 43 \\
\hline Aisselle & 9,1 & 2,1 & 10,2 & & - & - \\
\hline Poignet & & & & AR & & \\
\hline \multicolumn{7}{|l|}{ Fibulaire gauche (CEO) } \\
\hline Cheville & $\mathrm{AR}$ & AR & $A R$ & & 9 & \\
\hline \multicolumn{7}{|l|}{$\begin{array}{l}\text { Fibulaire gauche (tibial } \\
\text { antérieur) }\end{array}$} \\
\hline Tête de la fibula & 4,5 & 3,8 & 24,4 & & - & \\
\hline Fosse poplitée & 7,5 & 3,8 & - & & 10 & 33 \\
\hline \multicolumn{7}{|l|}{ Tibial gauche (CAH) } \\
\hline Cheville & AR & $A R$ & AR & & - & \\
\hline \multicolumn{7}{|l|}{ Facial gauche (nasal) } \\
\hline \multirow[t]{2}{*}{ Mastoïde } & 5,4 & 0,63 & 3,0 & & & \\
\hline & $\begin{array}{l}\text { Latence du } \\
\text { déclenchement, } \\
\text { ms }\end{array}$ & $\begin{array}{l}\text { Latence de } \\
\text { pointe, ms }\end{array}$ & $\begin{array}{l}\text { Amplitude du } \\
\text { potentiel au } \\
\text { déclenche- } \\
\text { ment, } \mu \mathrm{V}\end{array}$ & $\begin{array}{c}\text { Zone négative, } \\
\mathrm{ms}^{\star} \mu \mathrm{V}\end{array}$ & Distance, $\mathrm{cm}$ & $\mathrm{vc}, \mathrm{m} / \mathrm{s}$ \\
\hline \multicolumn{7}{|l|}{ Sites sensoriels } \\
\hline \multicolumn{7}{|l|}{ Médian gauche } \\
\hline Poignet-index & AR & AR & AR & AR & 14 & AR \\
\hline \multicolumn{7}{|l|}{ Ulnaire gauche } \\
\hline Poignet-auriculaire & $\mathrm{AR}$ & $\mathrm{AR}$ & AR & AR & 12 & $\mathrm{AR}$ \\
\hline \multicolumn{7}{|l|}{ Sural gauche } \\
\hline Latence mollet-malléole & AR & AR & AR & AR & 14 & AR \\
\hline \multicolumn{7}{|l|}{ Fibulaire superficiel gauche } \\
\hline $12 \mathrm{~cm}$ MMA & AR & AR & AR & AR & 12 & AR \\
\hline
\end{tabular}

Remarque : $\mathrm{AA}=$ abducteur de l'auriculaire, $\mathrm{AR}=$ absence de réponse, $\mathrm{CAH}=$ court abducteur de l'hallux, $\mathrm{CAP}=$ court abducteur du pouce, $\mathrm{CEO}=$ court extenseur des orteils, $\mathrm{EPI}=$ extenseur propre de l'index, MMA = malléolaire médian antérieur, $\mathrm{VC}=$ vitesse de conduction.

*La latence est le temps nécessaire pour que l'influx génère une réponse. En présence de démyélinisation, la latence est prolongée, comme l'illustrent les temps de latence pour le poignet (normale $<2,2 \mathrm{~ms}$ ) et le coude (normale $<4,4 \mathrm{~ms}$ ) au niveau du CAP médian gauche et pour le poignet au niveau de l'AA ulnaire gauche (normale $<2,2 \mathrm{~ms}$ ). L'onde $\mathrm{F}$ est une mesure de la conduction motrice pour le nerf entier. En présence de démyélinisation, l'onde F est prolongée, comme le montre l'AA ulnaire gauche.

\section{Discussion}

Le SGB est une polyneuropathie inflammatoire démyélinisante auto-immune. Nous décrivons le cas d'un homme de 63 ans qui a présenté un SGB environ 12 jours après avoir reçu sa première dose de vaccin ChAdOx1 nCoV-19. Il avait déjà fait un épisode de SGB 3 semaines après avoir reçu un vaccin contre la grippe saisonnière en 1999.

Le SGB serait causé par la destruction de la myéline et des membranes axonales des neurones périphériques médiée par des 
anticorps (IgG) et des cellules immunitaires (lymphocytes $\mathrm{T}$, macrophages). Un événement comme une infection, l'utilisation de médicaments (p. ex., inhibiteur des points de contrôle immunitaires), certains vaccins ou une chirurgie précède habituellement le syndrome. Même s'il n'y a pas de définition universelle du SGB imputable aux vaccins, la vaccination peut être pertinente si elle a été administrée jusqu'à 4 semaines avant le déclenchement des symptômes ${ }^{1}$. Toutefois, on ignore si la vaccination est un déclencheur réel du SGB. Une méta-analyse de 39 études a révélé une augmentation du risque relatif de SGB de 1,41 (intervalle de confiance [IC] à $95 \% 1,20-1,66$ ) après une vaccination antigrippale ${ }^{2}$, mais ces résultats n'ont pas été corroborés par la suite lors d'une grande étude rétrospective menée dans un seul pays au sujet du déclenchement du SGB dans les 180 jours suivant la vaccination ${ }^{3}$.

L'infection par le SRAS-CoV-2 aurait précédé certains cas de SGB; 73 cas ont été signalés dans 1 étude ${ }^{4}$. Nous avons dénombré plusieurs rapports de cas de SGB après la vaccination contre le SRAS-CoV-2, en lien avec les vaccins ChAdOx1 nCoV-19 (AstraZeneca) et BNT 162b2 (Pfizer) ${ }^{5-8}$.

Des rapports de cas antérieurs ont estimé le risque de récurrence du SGB à 1\%-6\% $\%^{9}$, comparativement à une incidence estimée de 1,11 cas par 100000 années-personnes ${ }^{1}$ dans la population générale. C'est donc dire que la probabilité de présenter un SGB peu de temps après la vaccination est plus élevée chez une personne qui a des antécédents de SGB. Lors d'une enquête, 126 patients ayant déjà présenté un SGB ont reçu un vaccin antigrippal, et aucun n'a présenté de récurrence de symptômes ${ }^{10}$. Pritchard et collègues ont constaté que chez 29 patients ayant reçu un vaccin dans les 6 semaines précédant un diagnostic initial de SGB, 2 (6,9\%, IC à 95\% 0,85\%-22,8\%) ont eu une récurrence de symptômes, généralement légers, après un autre type de vaccin administré subséquemment ${ }^{11}$. Ces résultats se fondent sur une enquête auprès des patients, et la confirmation d'une récidive de SGB n'a pas été documentée.

On dispose de peu de données probantes pour évaluer le risque de récurrence de SGB associé à la vaccination. Les CDC (Centers for Disease Control and Prevention) ont publié un guide en 2010 à partir des données tirées du Vaccine Adverse Event Reporting System et de la United Kingdom's General Practice Research Database, selon lequel les patients ayant présenté un SGB dans les 6 semaines suivant la vaccination antigrippale ne devraient pas recevoir d'autres vaccins antigrippaux s'ils ne sont pas exposés à un risque élevé de complications graves de la grippe ${ }^{12}$. En 2012, l'American Academy of Allergy, Asthma and Immunology a émis une recommandation similaire ${ }^{13}$. L'Agence de la santé publique du Canada stipule que la vaccination antigrippale est généralement contreindiquée chez quiconque a déjà manifesté un épisode de SGB dans les 6 semaines suivant l'administration d'un vaccin antigrippal, bien qu'il importe de soupeser le risque de SGB associé au vaccin et le risque de SGB associé à la grippe ${ }^{14}$. On ignore si cette recommandation est applicable à d'autres vaccins (p. ex., contre le SRAS-CoV-2) ou chez les patients ayant présenté une récurrence de SGB associée à la vaccination. Dans ces scénarios rares, les médecins doivent avoir une discussion approfondie avec leur patient au sujet du rapport risques:avantages en expliquant l'absence relative de données probantes et en tenant compte de la situation de chaque patient.

Aucune preuve convaincante n'a démontré que le vaccin contre le SRAS-CoV-2 provoque le SGB, et le présent rapport de cas n'établit pas un tel lien causal. Au Canada, la surveillance active des événements neurologiques, y compris le SGB, est assurée par le Programme canadien de surveillance active de l'immunisation, IMPACT, financé par l'Agence de la santé publique du Canada ${ }^{15}$. L'immunisation est une intervention vitale qui vise à réduire la transmission du SRAS-CoV-2 et les maladies qu'il cause; les contre-indications absolues sont rares.

\section{Références}

1. Shahrizaila N, Lehmann HC, Kuwabara S. Guillain-Barré syndrome. Lancet 2021;397:1214-28.

2. Martin Arias LH, Sanz R, Sáinz M, et al. Guillain-Barré syndrome and influenza vaccines: a meta-analysis. Vaccine 2015;33:3773-8.

3. Chen Y, Zhang J, Chu X, et al. Vaccines and the risk of Guillain-Barré syndrome. Eur J Epidemiol 2020;35:363-70.

4. Abu-Rumeileh $S$, Abdelhak A, Foschi M, et al. Guillain-Barré syndrome spectrum associated with COVID-19: an up-to-date systematic review of 73 cases. $J$ Neurol 2021;268:1133-70.

5. Patel SU, Khurram R, Lakhani A, et al. Guillain-Barré syndrome following the first dose of the chimpanzee adenovirus-vectored COVID-19 vaccine, ChAdOx1. BMJ Case Rep 2021;14:e242956. doi: 10.1136/bcr-2021-242956.

6. Hasan T, Khan M, Khan F, et al. Case of Guillain-Barré syndrome following COVID-19 vaccine. BMJ Case Rep 2021;14:e243629. doi: 10.1136/bcr-2021 $-243629$.

7. Trimboli M, Zoleo P, Arabia G, et al. Guillain-Barré syndrome following BNT162b2 COVID-19 vaccine. Neurol Sci 2021 Aug 4;1-2. doi: 10.1007/s10072-021-05523-5. [publication électronique avant impression].

8. McKean N, Chircop C. Guillain-Barré syndrome after COVID-19 vaccination BMJ Case Rep 2021; Jul 30;14(7):e244125. doi: 10.1136/bcr-2021-244125.

9. Basta I, Bozovic I, Berisavac I, et al. Recurrent Guillain-Barré syndrome - case series. Neurol India 2019;67:1536-8.

10. Kuitwaard K, Bos-Eyssen ME, Blomkwist-Markens PH, et al. Recurrences, vaccinations and long-term symptoms in GBS and CIDP. J Peripher Nerv Syst 2009; 14:310-5.

11. Pritchard J, Mukherjee R, Hughes RAC. Risk of relapse of Guillain-Barré syndrome or chronic inflammatory demyelinating polyradiculoneuropathy following immunisation. J Neurol Neurosurg Psychiatry 2002;73:348-9.

12. Fiore AE, Uyeki TM, Broder K, et al. Prevention and control of influenza with vaccines: recommendations of the Advisory Committee on Immunization Practices (ACIP), 2010. MMWR Recomm Rep 2010;59:1-62.

13. Kelso JM, Greenhawt MJ, Li JT, et al. Adverse reactions to vaccines practice parameter 2012 update. J Allergy Clin Immunol 2012;130:25-43.

14. Contraindications, precautions, and concerns: Canadian Immunization Guide. Ottawa: Government of Canada; 2020. Accessible ici : https://www.canada.ca/ en/public-health/services/publications/healthy-living/canadian-immunization -guide-part-2-vaccine-safety/page-3-contraindications-precautions-concerns. html\#p2c2t1 (consulté le 3 avr. 2021).

15. Bettinger JA, Halperin SA, Vaudry W, et al.; Canadian IMPACT members. The Canadian Immunization Monitoring Program, ACTive (IMPACT): Active surveillance for vaccine adverse events and vaccine-preventable diseases. Can Commun Dis Rep 2014;40(Suppl 3):41-4. doi: 10.14745/ccdr.v40is3a06. Accessible ici : https://www.canada.ca/en/public-health/services/reports-publications/canada -communicable-disease-report-ccdr/monthly-issue/2014-40/ccdr-volume-40-s -3-december-4-2014/ccdr-volume-40-s-3-december-4-2014-3.html (consulté le 10 avr. 2021). 
Intérêts concurrents : Sean Bagshaw déclare avoir reçu des subventions, des honoraires de consultation et autres de Baxter, indépendamment des travaux soumis. Il fait partie du Comité de surveillance de la sécurité des données pour l'étude I-SPY-COVID-19 et du comité consultatif de BioPorto. Aucun autre intérêt concurrent n'a été déclaré.

Cet article a été révisé par des pairs.

Les auteurs ont obtenu le consentement du patient.

Affiliations : Département de médecine, Faculté de médecine et de dentisterie (Ling, Bagshaw), Université de l'Alberta et Services de santé de l'Alberta; Département de médecine de soins intensifs et de médecine, Division de médecine interne (Villeneuve), Hôpitaux de l'Université de l'Alberta et des Sœurs Grises, Edmonton, Alb.

Collaborateurs : Ling Ling et Pierre-Marc Villeneuve ont analysé le dossier et les épreuves d'imagerie du patient et ont rédigé le manuscrit. Sean Bagshaw a révisé le contenu et analysé et interprété les données. Tous les auteurs ont révisé de façon critique le contenu intellectuel important du manuscrit; ils ont donné leur approbation finale pour la version destinée à être publiée et assument l'entière responsabilité de tous les aspects du travail.

Propriété intellectuelle du contenu : Il s'agit d'un article en libre accès distribué conformément aux modalités de la licence Creative Commons Attributions (CC BY-NC-ND 4.0), qui permet l'utilisation, la diffusion et la reproduction dans tout médium à la condition que la publication originale soit adéquatement citée, que l'utilisation se fasse à des fins non commerciales (c.-à-d. recherche ou éducation) et qu'aucune modification ni adaptation n'y soit apportée. Voir : https://creativecommons.org/licenses/by-nc-nd/4.0/deed.fr.

Correspondance : Pierre-Marc Villeneuve,

pierremarc.villeneuve@albertahealthservices.ca

La section Études de cas présente de brefs rapports de cas à partir desquels des leçons claires et pratiques peuvent être tirées. Les rapports portant sur des cas typiques de problèmes importants, mais rares ou sur des cas atypiques importants de problèmes courants sont privilégiés. Chaque article commence par la présentation du cas (500 mots maximum), laquelle est suivie d'une discussion sur l'affection sous-jacente (1000 mots maximum). La soumission d'éléments visuels (p. ex., tableaux des diagnostics différentiels, des caractéristiques cliniques ou de la méthode diagnostique) est encouragée. Le consentement des patients doit impérativement être obtenu pour la publication de leur cas. Renseignements destinés aux auteurs : www.cmaj.ca. 